\title{
Multi-Agent and Embedded System Technologies Applied to Improve the Management of Power Systems
}

\author{
A.Carrasco, M.C. Romero-Ternero, F. Sivianes, M.D. Hernández, J.I. Escudero \\ Departamento Tecnología Electrónica, University of Seville, Avda. Reina Mercedes $s / n$ 41012, \\ Seville, Spain \\ acarrasco@us.es,mcromero@dte.us.es,sivianes@dte.us.es,marilohv@dte.us.es,ignacio@us.es \\ doi: 10.4156/jdcta.vol4.issue 1.8
}

\begin{abstract}
This article explores a number of improvements made on Supervisory Control and Data Acquisition (SCADA) systems which allow them to be successfully used for automated surveillance. Even telecontrol operators who have limited experience with computers were able to employ the system without any difficulties. Other advances made by taking advantage of the strongest features of embedded and multi-agent system technologies are also featured in this article. These developments have been tested in a true industrial environment. Positive results and feedback have been provided by the tests.
\end{abstract}

\section{Keywords}

Multi-agent systems, embedded systems, telecontrol, surveillance, SCADA.

\section{Introduction}

Currently it is inconceivable to have a domestic or industrial area without a continuous supply of electric energy. A good part of the comfort we enjoy in our homes, as well as a good part of industrial work output, depends on constant electric supply. Therefore, it is absolutely necessary to guarantee a continuous and non-altering supply of electric energy at all times. In order for this supply to satisfy the needs of its users, the installation of a complex control and management device is required. Telecontrol allows this control and management to be carried out, and is therefore is a very important mechanism which should be improved as much as possible to take advantage of the advances in new technology, so that the decisions made in each moment are strongly supported by the most efficient telecontrol mechanisms.

The main aim of SCADA systems involves the supervision and remote control of devices, mainly sensors and actuators, which can be found in isolated and remote places [1]. Information that these devices provide, for example the measurement of the intensity of an electric current on a power line, involves short information which is no longer than a few dozen bytes. As a result, technology that was traditionally used is still adequate today for the management of the device's data, and will probably continue to be used in the future.

Nevertheless, the transmissions and network technologies are not the only fields that have spectacularly evolved over the last few years. Techniques involving information compression and the use of multimedia signals have also experienced notable advances recently. This has been brought about due to the modern day use of digital audio and video, which can be efficiently processed by PC systems at low cost to obtain good visual results of this information by using relatively low speeds to send the bits. Codecs such as MPEG-4 allow for the compression of high resolution video signals at less than $1 \mathrm{Mbps}$ [2].

The man-machine interface, which is used to make the management of the SCADA network operation simpler, normally indicates the current state of the information as a chain of text, and, in some cases, with a symbol or color that signals the current state of the device. By adding audio and video multimedia information, the management of the system is improved by the operator, mainly in remote facilities where no stable support staff is available. This improves the management of big SCADA systems [3].

The use of embedded systems and multi-agent technology, in which the sensor network is used as a means to compile physical data about the system, including visible and thermal audio and video, are highly useful and allow for automatic execution. This information is compiled to be sent to a central node or base station where it is displayed, stored, treated, and sent to superior nodes in the remote management network.

\section{Multimedia in SACADA Systems}

Rather than talking about integrating multimedia data into SCADA systems, it would be better to think about integrating SCADA data into modern network systems.

Typical transmission links and protocols used on SCADA networks do not fulfil the bandwidth requirements that multimedia data deployment impose, as data acquired from SCADA devices is usually transmitted over low-bandwidth links using serial protocols. 
Another important key factor is that temporal requirements on the reception of the acquired data are very tight on SCADA systems; cycle time on SCADA buses generally ranges between $10 \mathrm{~ms}$ and $100 \mathrm{~ms}$. Furthermore, transmission reliability is also very important as loss of data might cause a big impact to the integrity of the system.

We have successfully injected SCADA data into an Ethernet system, using a custom SCADA bridge (Figure 1). Data transmitted using the IEC870 protocol from a Multitrans PLC device - which gives data about the voltage and intensity of a power line, among other features- is injected into an Ethernet network using bidirectional serial-to-Ethernet converters [4].

The converter we have used, named the IS-Server device, takes serial data from the Multitrans device and converts it into TCP packets. It sends them to a predefined IP network address. If the control system needs to receive data in its original serial form, the inverse process may be applied using another IS-Server device.

In our tests, status data, which is periodically polled from a Multitrans device, travels between two Ethernet-based LANs connected via a $100 \mathrm{Mbps}$ fiber optic link, resulting in the reception of the SCADA data in less than $10 \mathrm{~ms}$, even when a raw multimedia stream, which needs nearly all the available bandwidth, was also sharing the fiber optic link.

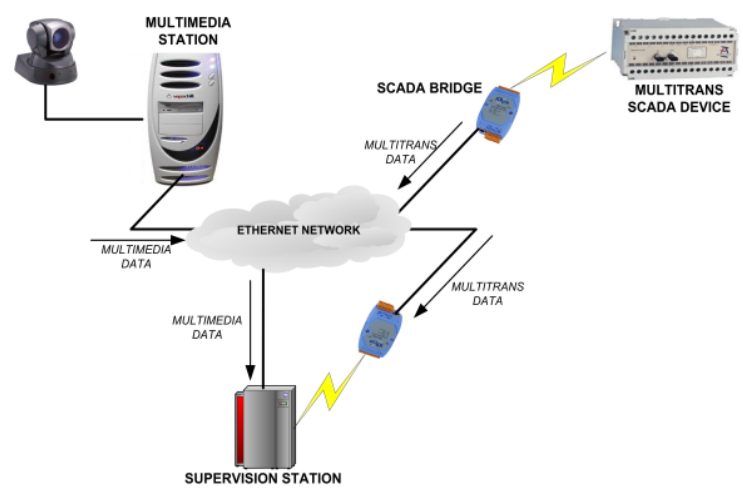

Figure 1. Ethernet SCADA bridge.

As proven above, from the time point of view, sharing the physical medium between SCADA and multimedia data is possible in Ethernet networks, but transmission reliability must also be considered. Ethernet hub devices use CSMA-based medium sharing techniques, which can lead to packet loss in a high traffic scenario, due to packet collision [5]. Therefore, switches should be the starting point in the design of any multimedia SCADA system.

Network management techniques such as Quality of Service might also be used to ensure SCADA data always gets the bandwidth it needs [6]. QoS allows for packet prioritization and bandwidth provision, based on one or more factors, such as the IP address of the sender.

\section{IDOLO System Structure}

\subsection{Interface}

The IDOLO interface has been built as a webbased software system, supported by a database system, which manages all needed system configuration, and allows operators to easily have access to all multimedia information of the SCADA system.

Being web-based means it can be independently run from an existing SCADA/HMI system, and at the same time it can be integrated into HMI systems supporting web navigation. HMI software supporting ActiveX technology can easily be integrated into the IDOLO software system via an embedded ActiveX web browser.

Furthermore, some web browsers, such as Internet Explorer, also support ActiveX components; so software components specific to the IDOLO system have been developed as ActiveX controls.

The IDOLO navigation system has been developed using PHP programming language on the server side, and Javascript on the client side. PHP is used to control the content and layout of served HTML pages, and to serve as the interface between the IDOLO system and a MySQL database server that holds all system configurations, such as data about cameras or the design of multimedia synoptics of each station. Using Javascript permits interfacing with web-browser, allowing the IDOLO system to be informed of user input events (e.g. mouse clicks) or control specific browser properties.

\subsection{Camera Management}

From a logical point of view, the IDOLO system is made up of 2 elements: stations and cameras. It's hierarchically organized so that stations own one or more cameras.

Three types of cameras have been used. Each one has special features, which has led to the use of different approaches to manage each camera type.

First, network cameras, which do not include internal storage systems, need an external system to store their video. The network camera chosen, the AXIS 230, uses MJPEG codec, which is no more than a sequence of JPEG images. MJPEG is not an efficient video codec, as it only performs spatial compression (based on Discrete Cosine Transformation), as opposed 
to MPEG-4 that performs both spatial and inter-frame compression. As it needs an external FTP server with enough storage space to stock the video, this can make for an excessive use of network bandwidth.

Webcams and analog cameras need dedicated PCs to perform the needed compression and streaming. We have chosen the Windows Media software platform to deal with these processes, which allows for full control over nearly all aspects regarding the previously mentioned procedures.

- Windows Media Encoder is a software component that takes video and audio from USB cameras and frame grabbers and compresses them with Windows Media codecs, variants of MPEG4 codec in the case of video and MP3 in the case of audio. It also allows for the immediate storage of compressed content in both local and remote storage.

- Although WME also allows for the use of streaming by using HTTP/TCP protocols, Windows Media Services is a specialized component that takes data compressed with WME and pushes it to the network using RTSP/UDP protocols. Apart from using more efficient network protocols for streaming, WMS is the necessary solution for allowing external stations to receive multimedia content from WME encoders, when the IP addresses of the encoder stations are inside the local scope.

\subsection{Multimedia synoptics}

HMI systems typically use synoptics to show, in a single screen, all the representative data about SCADA elements. The IDOLO system takes this approach and adapts it to the multimedia field (Figure 2) by:

- Showing video and audio coming from cameras located at a given station.

- Providing graphics of all representative elements in a station, which, when clicked, command the PTZ cameras to move to a preset position so they focus on the selected element.

- Allowing for the activation of manual control on PTZ cameras, which are able to control them using the mouse or even a joystick.

Multimedia synoptics also provide a solution to the inherent delay introduced by the MPEG-family codecs, which can make the manual control of PTZ devices a bit confusing as images run late in respect to camera movements.

\section{Deployment in an Electrical Facility}

The final goal of the IDOLO project has been the integration of our system into a real, working SCADA system. For this reason, we have garnered the cooperation of the Medina Garvey electrical facility, a regional electrical provider located in Seville.

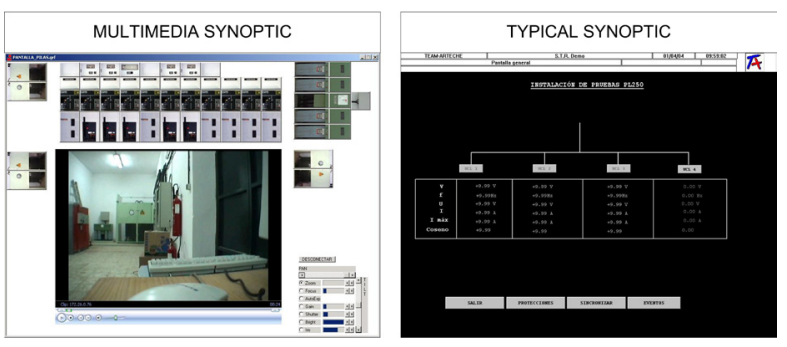

Figure 2. Screenshots comparing multimedia synoptics and classic synoptics.

The existing facilities owned by Medina Garvey have been controlled and supervised using SCADA hardware and software developed by Team Arteche. An example of those SCADA components has been the Multitrans device used in the integration tests.

To avoid compromising the integrity of existing system, it was decided that separate links for sending multimedia and SCADA information from remote stations to the supervision station would be used. The modern facilities in Medina Garvey were equipped with fiber optic links to communicate with the supervision station, so spare fiber cabling was used for these purposes. These fiber optic links are more than adequate for transmitting high-quality multimedia streams.

There are some older facilities that do not use such high-speed links. In such cases, serial radio links which do not have enough bandwidth to send multimedia information have been used to transmit SCADA status information. Yet these facilities were also equipped with telephone lines, so ADSL technology was used for sending our multimedia streams. However, using ADSL limited the transmission to fewer, and lower-quality streams. We have tested the transmission of one $512 \mathrm{Kbps}$ stream, and alternatively, two $256 \mathrm{Kbps}$ streams, successfully.

The SCADA/HMI system used by Medina Garvey, which is a SIPCON HMI system, is a private system which does not allow for the integration of external components. Therefore, IDOLO software has been deployed separately, by using a PC with an Internet Information Services web-server to provide web content to the intranet.

\section{Improvement Found by Using Embedded and Multi-Agent Technologies}

When this system was installed in a real-life environment, we interviewed operators in order to find out about their level of satisfaction. One of the improvements that they proposed was to make IDOLO 
system a black box for them, meaning a system which is easy to maintain and configure just by pressing a button, without needing any experience with computers. This led us to consider the possibility of developing the system by using embedded technology.

Embedded systems shouldn't be thought of as PCs with general characteristics. Even though it is becoming more and more possible to replace them in some applications, due to the development of microprocessors and microcontrollers.

Embedded systems could be considered hard - soft applications, which make them more interesting as far as the immediate future of the Information and Communications Technology sector [7][8]. The main idea is to use systems which are practically invisible and made up of microprocessors and software in very small systems. These systems allow us to obtain information and process it anywhere, in a quick and easy way (ubiquitous computation). If we are able to create a network composed of these embedded systems, which are almost invisible, and we provide them with communication and action abilities, we can create a spatially distributed hardware structure that is available at all times. This allows for the user to be able to anticipate things (pervasive computation) (Marwedel, 2003) (Sutter, 2003). All of this can be accomplished in a more secure and reliable way, with quick and easy maintenance and lower economic costs and electrical consumption seen when using traditional PCs.

By introducing these systems in hierarchical schemes of "surveillance" distributed in substations or transformation centers, we can accomplish an automatic surveillance which allows for preventive maintenance operations. In this way we can anticipate possible anomalies that could produce blackouts, which are quite problematic for the end users and, as a result, have significant economic impact on the electric utilities.

When talking about automatic surveillance we are referring to not only proactive but also reactive surveillance. This implies that the elements which carry out the surveillance have human capacities and are able to make decisions and act by themselves. This human behavior has to be programmed, and we are working to achieve this by using multi-agent system (MAS) technology.

MAS are systems composed of multiple interacting computing elements, known as agents. Agents are computer systems with two important capabilities [11]:

- They are capable of autonomous actions and can decide by themselves.

- They are capable of interacting with other agents in a social way (cooperation, coordination, negotiation...)
As a result, different interactions among the different kinds of agents are being modeled. Each defined agent is responsible for the surveillance in a specific way because each of them has a fixed intelligence level. Some of them have less capacity to process, so they can only carry out a basic analysis of captured situations, whereas, others have more processing capacity, so they can accomplish an advanced analysis.

What kind of information is going to be analyzed? We are working with several types of sensors (senses in Figure 3) which capture image (visible and infrared), smoke and volumetric measurements. Depending on the kind of data, the analysis is carried out in a different way. After modeling the interactions, we are going to design the MAS. The main objective is to cover the maximum amount of elements in the electrical facility environment (surveillance targets) with the minimum agents, while keeping agents in cooperation at the same time. Autonomy and learning capacities are very important for our system, and both of them are great advantages that MAS provide.

\section{Architecture of the New System}

Two levels of surveillance are used. In the lowest hierarchical level there is a network composed by guard agents which don't need to have an excessive capacity to process. These vigilant systems are always collecting information (through sensors) and they have to control their environment by generating alarms or with corrective actions (actuators) if appropriate. They have to interact with their environment in a quick and easy way, and processing in real time. They have certain mobility in a limited area.

Traditionally, these reactive systems, which are in continuous interaction with the environment, execute certain steps according to that environment, and wait for events to happen. When this occurs, they carry out the process operations by generating out data and changing it to a new state. In this way, anticipation or prevision capacity is limited, since it is restricted to a series of events from a series of known states, leading the system to another waiting state.

However, if we make these guards interact, we can create a knowledge network. This network allows guards to request information by searching for the most adequate solution. If it is not possible to find a solution, our system works by acceding to the highest level, where there is a supervisor with a higher computation capacity. This can transmit the solution to the lower level, or request information from other supervisors. [13].
The high-level architecture is shown in Figure 3 


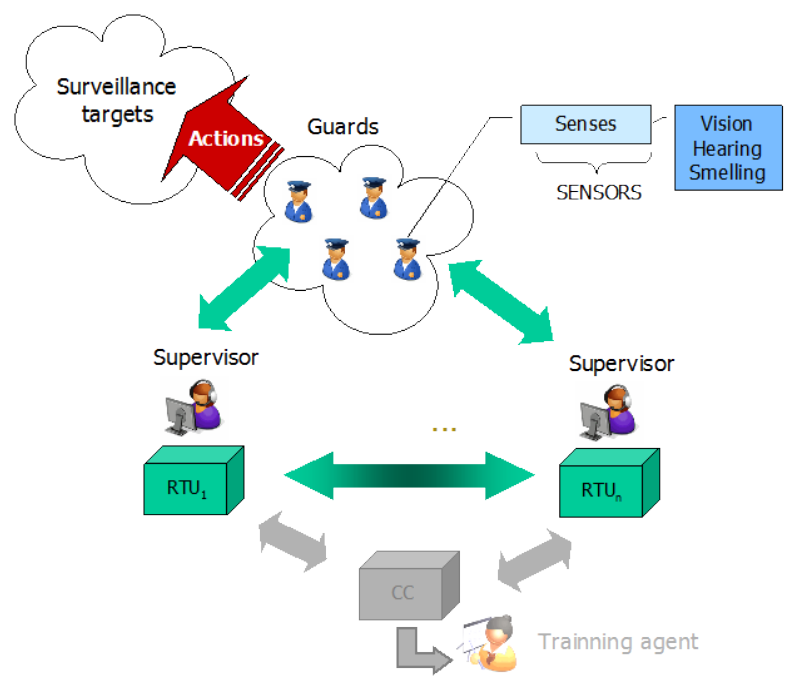

Figure 3. Scheme for the new system.

\subsection{Description of the platform hardware used}

The guard agent on the lower level has been implemented with an embedded system, based on SquidBee [9].

SquidBee developed by Libelium (spin-off of the University of Zaragoza), has the haracteristic of the first "open source mote" [10].

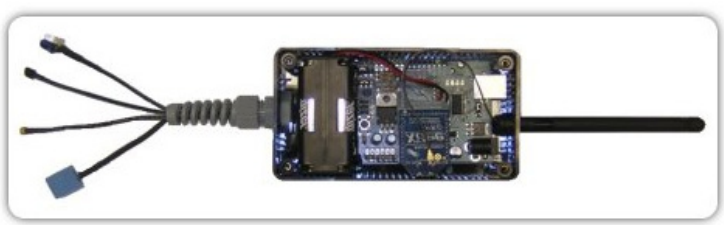

Figure 4. SquidBee mote.

It's characteristics are:

- Arduino control module.

- Wireless communication module, Xbee (ZigBee).

- Open source mote.

- $9 \mathrm{~V}$ feed battery.

- $12 \mathrm{I} / \mathrm{O}$ pin digitals.

- 6 pin of analogic inputs.

- 5 PWM pin of analogic otput.

- USB connection to PC (Windows, Linux and Mac compatable).

- Sensors of: Temperature, Humidity, Light levels and posibility of easily adding new sensors.
- Topology of Peer-to-peer, point-to-point, point-to-multipoint and mesh networks. Possibility of directing up to 65,000 motes.
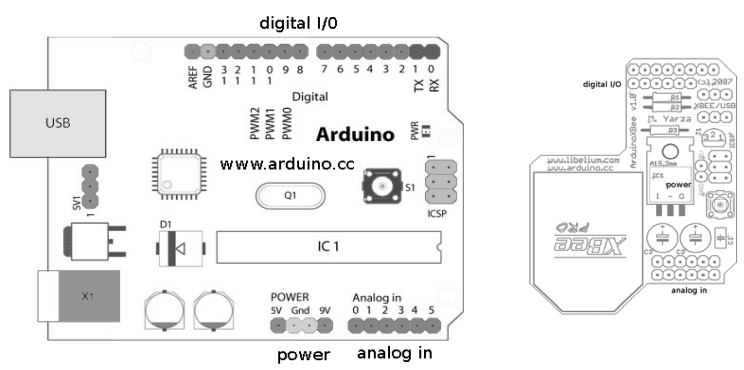

Figure 5. SquidBee map.

Meshlium, an embedded system developed by Libelium, has been selected to the "supervisor" agent. Meshlium specifications are:

- $233 \mathrm{MHz}$ AMD Geode SC1100 CPU (fast 486 core).

- 64 or 128 MB SDRAM.

- Operating system and application stored on CompactFlash card.

- About 3 to $5 \mathrm{~W}$ at $12 \mathrm{~V}$ DC.

- Three front panel LEDs, one pushbutton switch, can be controlled through CPU GPIO pins. Console I/O redirected to serial port.

- LPC bus for adding serial ports, ISA style I/O, GPIO etc. I2C bus for user interface, software lock devices etc.

- 1 to 3 Ethernet channels (National DP83816), 2 or 1 miniPCI slots, 1 serial port (console)

- $1.2 \mathrm{Kg}$ weight (without the antenas).

- 1-2 minutes: time in which all services run

The heart of Meshlium. These Meshlium teams are based on systems of building PC Engines which provide cost effective added hardware value with SBC (Single Board Computer) to the processing and development network, including wireless routers, firewalls, VPN, industrial Ethernet devices, or other general networking devices.

Its $\mathrm{CPU}$ is $233 \mathrm{MHz}$, and it has an AMD Geode SC1100 CPU (fast 486 core) with 64 to $128 \mathrm{MB}$ of SDRAM. The operative system and applications are stored in a CompactFlash card. It consumes around 3$5 \mathrm{~W}$ to $12 \mathrm{~V}$ DC (excluding the miniPCI cards which can be added). The feed can be supplied by a jack or thrugh an Ethernet PoE (Power over Ethernet) cable. It accepts a tension range between $+7 \mathrm{~V}$ y $+18 \mathrm{~V}$ DC. An LPD expansion bus is available to add series docks, GPIO and a I2C bus for user interfaces. Additionally, it includes a button and three leds which provide 
information on the current states. It features 1 to 3 ethernet channels (National DP83816), 2 or 1 slot miniPCI and a dock with a console.

The WRAP microcontrol panel includes the tinyBIOS PC Engines in the panel, in version 1.11. The operating system that it includes is a distribution of linux, supported in the 2.6 kernel, and based on WRAP specific distribution and voyage linux, which is based on Debian. The figure 7 features a diagram of system blocks.

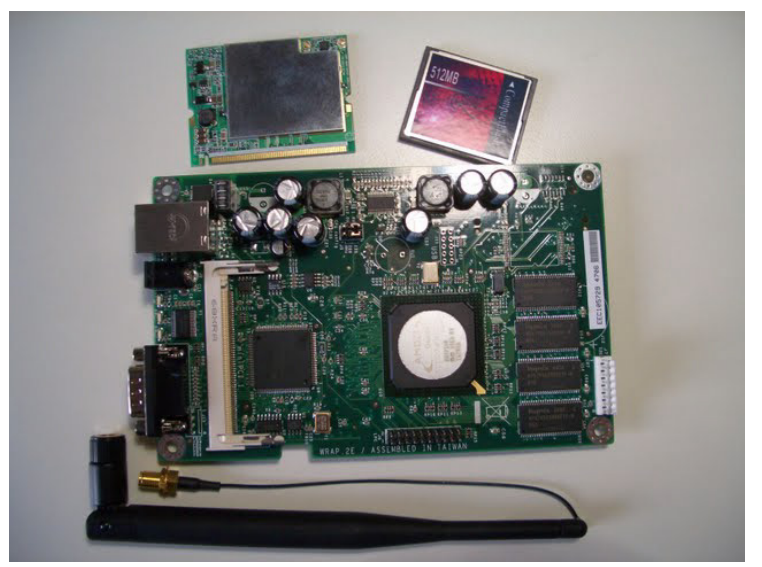

Figure 6. Meshlium card.

\section{Pilot Test}

This chapter describes our experience during a pilot test carried out on the previously described platform hardware and based on the PCEngine panels.

This pilot test was done in collaboration with the Technology of Materials research group of the University of Cadiz (Spain). This group specializes in the application of multiagent systems in environments with automated production systems.

We took advantage of this group's experience with agents in the production process and used an agent in the test which automatically managed of the engineering project [12], which could be seen as an endorsement of a professional association. Initially it may seem that the topic was diverted (to electric facility control), although our true interest is the execution of an actual agent that is as refined as possible, and in our demand to use resources similar to those that the guard and supervising agents proposed, which was the main motivation behind this decision [14], [15].

Keeping in mind that the main objective was to test the sturdiness and flexibility of the chosen support hardware, we though it would be convenient to carry out this trial both in free use and private environments by executing a pilot test in a Microsoft (Windows XP
SP3) platform and another with S.O. Linux (Ubuntu 9.04). The test involved installing and executing the agents in a machine with limited resources, while entering data to correctly verify the functioning actions.

In the case of the Microsoft platform, since there was a great deal of memory and capacity restriction, the S.O. load had to be decreased by eliminating unnecessary S.O. applications, such as games, etc. Execution was completed after many failed library attempts. It has been ruled out that these failures are cause to use the hardware resources, since the original resources proved sufficient. After many tests, it was determined that the failures were due to an inappropriate .NET version. This was resolved by appropriately adjusting the correct. NET for this hardware platform.

The experience in the Linux platform turned out to be more bearable. Keeping in mind the imposed hardware restrictions, the results are considered to be successful upon obtaining the execution of the agents of both platforms (although each had a different level of difficulty).

It can therefore be concluded that our research confirms the possibility of using hardware embedded systems with few resources for the field execution of specific tasks (with agents) which are transparent for the final user.

\section{Conclusions}

This article describes the IDOLO system which provides multimedia information in the HMI/SCADA system for the telecontrol of electric networks. It has also detailed how this system functions by using embedded systems, which cause improvements. This system has successfully been tested at a real electric company allowing for the operators of this company to be provided with multi-media information from remote stations. This has made for faster and more complete system management, and made it no longer essential for staff to travel out to remote areas if unnecessary, due to the use of live images of the maintenance work which allow for long-distance inspection of remote facilities, etc. This information is available in the control centers and at people's homes through the internet.

When multi-agent technology takes advantage of autonomous capacities and cooperation among different agents, the system is provided with intelligence and automation that allows for an operator to receive information selected by the system itself. 


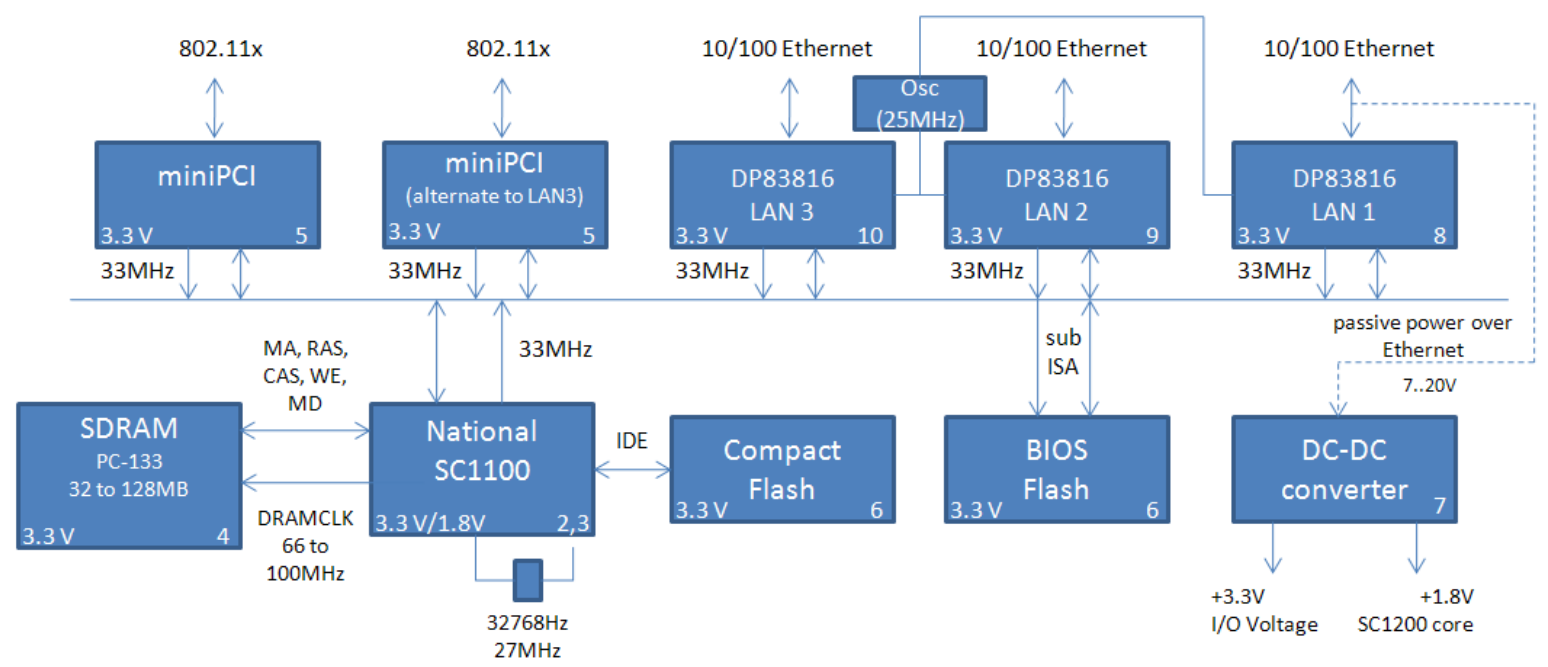

Figure 7. Meshlium architecture description.

An automatic surveillance system has been developed which uses analysis techniques with infrared and visible image processing, along with the information provided by other sensors, to improve and facilitate the telecontrol tasks of the operators located in the control centers.

\section{Acknowledgements}

The work described in this paper has been funded by the Ministerio de Ciencia y Tecnología within I + D + I National Program through the project with reference number TEC2006-08430, and by the Junta de Andalucía through the project with reference number P08-TIC-03862.

We would also like to thank the Material Technology Research Group of Cadiz University, ISIS Engineering (Seville) for providing us with ISSERVER prototypes, and Medina-Garvey electrical company for letting us use their facilities.

\section{References}

[1] Boyer, S. A., 1999. SCADA: Supervisory Control And Data Acquisition, $2^{\text {nd }}$ Edition, ISA - The Instrumentation, Systems and Automation Society, New York (United States).

[2] ISO/IEC, 1999. Information technology - Coding of audio-visual objects - MPEG-4, ISO/IEC 14496.

[3] Romero, M. C., Díaz, S., Sánchez, G., Escudero, J. I., 2004. SIP Application To Multimedia Telecontrol Of Power Systems. In World Automation Congress Proceedings, pp. $131-137$.
[4] IEC, 1990. Telecontrol and equipment systems - Part 5: Transmission protocols, IEC 60870-5.

[5] ISO/IEC, 1993. Information technology - Local area networks - Part 3: Carrier sense multiple access with collision detection, ISO/IEC 8802-3.

[6] Arindam, P., 1999. QoS in Data Networks: Protocols and Standards. Ohio State University, http://www.cs.wustl.edu/ jain/cis7889/ftp/qos protocols/ index.html.

[7] Marwedel, P., 2003. Embedded System Design. Springer, ISBN: 978-1-4020-7690-9

[8]Sutter, E., 2002. Embedded System Firmware Demystified. Publishers Group West, ISBN 9781578200993.

[9] Libelium home, http://www.libelium.com

[10] Atmel Home, http://www.atmel.com/products/avr32/

[11] Wooldridge, M., 2002. An Introduction to MultiAgent Systems. Wiley, ISBN 047149691.

[12] Aguayo, F., 2007. Sistemas Avanzados de Fabricación Distribuida. Ed. Ra-Ma, ISBN 978-84-7897-804-5.

[13] B. Sasikumar, V. Vasudevan, 2008. An agent based TCP/IP for Wireless Networks, JDCTA: International Journal of Digital Content Technology and its Applications, Vol. 2, No. 3, pp. $47 \sim 52,2008$.

[14] Yazed Al-Sawi, Ajlan Al-Ajlan, Khalid Al-Drewiesh and Abdullah Bajahzer, 2009. An Efficient Search Agent Software for Multi-Agent Systems, JDCTA: International Journal of Digital Content Technology and its Applications, Vol. 3, No. 1, pp. $73 \sim 87,2009$.

[15] Romero, M.C.; Sivianes, F.; Carrasco, A.; Hernandez, M.D.; Escudero, J.I. Managing emergency response operations for electric utility maintenance. Industrial Electronics Magazine, IEEE. Vol. 3, Issue 3, pp. 15-18, Sept. 2009. 